\title{
Bioactive Potential of Brazilian Plants Used as Food with Emphasis on Leaves and Roots
}

\author{
Fernanda L. B. Mügge, Sarah M. S. Prates, Juliana de Paula-Souza, \\ and Maria G. L. Brandão
}

\footnotetext{
F. L. B. Mügge

Centro Especializado em Plantas Aromáticas, Medicinais e Tóxicas (Ceplamt), Museu de História Natural e Jardim Botânico, Universidade Federal de Minas Gerais (UFMG), Belo Horizonte, Brazil

Zentrum für Molekulare Biologie, Universität Heidelberg, Heidelberg, Germany

\section{S. M. S. Prates}

Centro Especializado em Plantas Aromáticas, Medicinais e Tóxicas (Ceplamt), Museu de História Natural e Jardim Botânico, Universidade Federal de Minas Gerais (UFMG), Belo Horizonte, Brazil

Programa de Pós-Graduação em Ciências de Alimentos, Faculdade de Farmácia, Universidade Federal de Minas Gerais (UFMG), Belo Horizonte, Brazil

J. de Paula-Souza

Centro Especializado em Plantas Aromáticas, Medicinais e Tóxicas (Ceplamt), Museu de História Natural e Jardim Botânico, Universidade Federal de Minas Gerais (UFMG), Belo Horizonte, Brazil

Departamento de Botânica, Centro de Ciências Biológicas, Universidade Federal de Santa Catarina (UFSC), Florianópolis, Brazil
}

M. G. L. Brandão (西)

Centro Especializado em Plantas Aromáticas, Medicinais e Tóxicas (Ceplamt), Museu de História Natural e Jardim Botânico, Universidade Federal de Minas Gerais (UFMG),

Belo Horizonte, Brazil

e-mail: mbrandao@ufmg.br 


\section{Introduction}

Archaeological finds show that plants have been used for millennia as sources of food and medicine in the Americas. Colonizing Europeans indeed learned the use of species such as corn, potatoes, cassava, tomatoes, cocoa, avocado, pineapple, and cashew, from the Amerindians (Maezumi et al. 2018). Native plants continue to be widely used in Brazil, but the intense miscegenation of cultures that occurred over recent centuries has also popularized the use of exotic species (Ferrão et al. 2008). The intense industrialization and urbanization of the country, which started in the 1950s, has deprived the Brazilian population of knowledge about the potential of native plants for use as food and medicine (Domingues et al. 2012; Fernandes 2004). These processes were aggravated by different economic cycles, starting with the exploitation of brazilwood (Paubrasilia echinata (Lam.) Gagnon, H.C.Lima \& G.P.Lewis, Fabaceae) by the Portuguese. The economy is currently dominated by mining and agribusiness, both of which seriously damage native natural ecosystems (Dean 1996). Today, only $7 \%$ of the Atlantic Forest territory remains, while other ecosystems such as the savannas (Cerrado) and dry forests (Caatinga) are rapidly being replaced by monocultures of eucalyptus, sugarcane, soybeans, and livestock (Bockmann et al. 2018; Venâncio et al. 2018). As a consequence, not only knowledge but also the native plant species themselves are being lost (Brandão et al. 2008a, b; Mügge et al. 2016).

In contrast to this scenario, the consumption of healthier food and the transition to plant-based diets are growing in the developed world (Kahleova et al. 2017; Melina et al. 2016). The development of a "bioeconomy", based on the sustainable use of biodiversity with less impact on the environment is also coming into favor (Valli et al. 2018), and traditional knowledge concerning the use of plant species used as food can be very valuable in this context. Information on species used by local populations in the past can reveal the existence of plants with the potential to be used as functional foods or used as a source of purified biological products. To contribute to this aim, during the last decade, our research group has focused on recovering traditional information about useful Brazilian plants from manuscripts and books published prior to 1960. Each work is carefully searched for data regarding the use of plants, and the information is then publicized (Brandão et al. 2011; Brandão et al. 2012; Breitbach et al. 2013; Fagg et al. 2015; Teixeira et al. 2019). Furthermore, the information is entered into an online database named Dataplamt (www.ceplamt.org.br/dataplamt), built to organize and make information readily available. Searches can be done through links referring to popular and scientific names, traditional uses, places where the plant occurs, and full texts extracted from original books or documents. To date, Dataplamt contains information from 66 documents on 3400 species of Brazilian useful plants.

In two previous studies, we have focused on the search for data concerning native food species. Oliveira et al. (2012) studied the native plant foods recorded in field books of 16 European naturalists who traveled through Brazil in the nineteenth century. More recently, Teixeira et al. (2019) showed data on 504 edible fruits 
recovered from the book "Dicionário das plantas úteis do Brasil e das exóticas cultivadas" (Dictionary of Useful Plants of Brazil and Exotic Cultivated), organized by the Portuguese botanist Manoel Pio Corrêa (1874-1934), in 1926. In this study, we present additional data about native plant species used as food, with emphasis on leaves and roots, recovered from books published by nine authors from the sixteenth to the twentieth centuries.

\section{Methods}

\subsection{Search and Organization of Data}

Firstly, we did a search in Dataplamt for plants that were classified as edible ("comestível," in Portuguese). Then, we searched the data using the key words: "frutos comestíveis" (edible fruits), "raízes comestíveis" (edible roots/tubers), "folhas comestíveis" (edible leaves), "flores comestíveis" (edible flowers), and " brotos comestíveis" (edible sprouts)". Information from books published by nine authors, living in different regions of Brazil from the sixteenth to the twentieth centuries, were selected. Table 1 shows the names and nationalities of each author, year of publication of their books, the area upon which their studies focused, and the numbers of edible plant species recorded by each one.

Table 1 Authors' nationalities, phytogeographical domains and regions studied in Brazil, years of the studies and number of plants cited in each publication

\begin{tabular}{l|l|l|l|l}
\hline $\mathrm{Abb}^{\mathrm{a}}$ & Author/nationality & Area of study & $\begin{array}{l}\text { Period in } \\
\text { Brazil }\end{array}$ & $\begin{array}{l}\text { Number of } \\
\text { plants }\end{array}$ \\
\hline SO & $\begin{array}{l}\text { Gabriel Soares de } \\
\text { Sousa/Portuguese }\end{array}$ & Atlantic Forest (Bahia state) & $1575-1592$ & 5 \\
\hline LI & $\begin{array}{l}\text { Friar Cristovão de } \\
\text { Lisboa/Portuguese }\end{array}$ & Amazonia (Maranhão state) & $1624-1635$ & 5 \\
\hline PI & Guilherme Piso/Dutch & $\begin{array}{l}\text { Atlantic Forest and Caatinga } \\
\text { (Pernambuco state) }\end{array}$ & $1637-1644$ & 7 \\
\hline VE & $\begin{array}{l}\text { Friar Mariano } \\
\text { C. Vellozo/Brazilian }\end{array}$ & $\begin{array}{l}\text { Atlantic Forest and Cerrado (Minas } \\
\text { Gerais and Rio de Janeiro states) }\end{array}$ & Natural & 18 \\
\hline SP & $\begin{array}{l}\text { Richard Spruce/ } \\
\text { English }\end{array}$ & Amazonia (Amazonas and Pará state) & $1849-1864$ & 4 \\
\hline CH & $\begin{array}{l}\text { Pedro Napoleão } \\
\text { Chernoviz/Polish }\end{array}$ & All regions of Brazil & $1840-1855$ & 9 \\
\hline PK & $\begin{array}{l}\text { Theodor Peckolt/ } \\
\text { German }\end{array}$ & All regions of Brazil & $1847-1912$ & 30 \\
\hline CR & $\begin{array}{l}\text { Manoel Pio Corrêa/ } \\
\text { Portuguese }\end{array}$ & All regions of Brazil & $\begin{array}{l}18 X X- \\
1934\end{array}$ & 26 \\
\hline CN & Paul Le Cointe/French & Amazonia & $1891-1956$ & 96 \\
\hline
\end{tabular}

${ }^{\mathrm{a}}$ Abbreviation 
To check whether a species was native of Brazil, as well as the current botanic name, was performed using the website "Flora do Brasil". ${ }^{1}$ It is often difficult to identify species from books published between the sixteenth and eighteenth centuries, because taxonomy as we currently know it was established only in the late eighteenth century, and the rules of botanical nomenclature did not exist until the beginning of the twentieth century. In particular, imprecise identification of some of the plants referred by Sousa (1587), Piso (1648), and Lisboa might detract from the accuracy of the study. However, reliability is somewhat increased by the fact that the editors of each of these books have added comments and more precise identification of the described species. Data on recent laboratory studies on bioactivity were obtained from PubMed.

The recorded plants were then divided in two tables: one with species submitted to studies that confirmed its health benefits (Table 2) and another table with the other plants that have not been studied (Table 3). Information about which author cited the plants is also included in the Tables (abbreviated names), as well as citations by other authors also present in Dataplamt.

\subsection{Species Cited by Other Authors}

The results obtained from this study were compared with data recorded by European naturalists who lived or travelled in Brazil in previous centuries. The first search was performed in Oliveira et al. (2012), which describes historical data recovered from the nineteenth century (indicated as "1-167" in Tables 1 and 2). Other data were obtained directly from the field books of the naturalists Auguste de Saint-Hilaire (Brandão et al. 2012), George Gardner (Fagg et al. 2015), and Richard Spruce (Santos-Fonseca et al. 2019). Additional data collected by von Martius in Amazonia in the nineteenth century was recorded by Breitbach et al. (2013) and from Corrêa in the twentieth century in Teixeira et al. (2019). Importantly, these naturalists provide detailed information about the plants and its uses. They also provide an adequate coverage of the country in terms of area and phytogeographic domains: von Martius, for example, described plants from the North region (Amazon); Gardner and Burchell from Midwest region (Caatinga and Cerrado); and Saint-Hilaire described species from Southeast and South regions (Cerrado and Atlantic Forest).

\footnotetext{
${ }^{1}$ See at: http://floradobrasil.jbrj.gov.br/2010/
} 
Table 2 Food species cited by the authors submitted to bioactivity studies

\begin{tabular}{|c|c|c|c|c|c|c|c|c|c|c|c|}
\hline $\begin{array}{l}\text { Family/species/ } \\
\text { popular names }\end{array}$ & & $\mathrm{SO}$ & LI & PI & VE & SP & $\mathrm{CH}$ & PK & $\mathrm{CN}$ & $\mathrm{CR}$ & $\begin{array}{l}\text { Correlated studies on } \\
\text { bioactivity }\end{array}$ \\
\hline $\begin{array}{l}\text { Aizoaceae } \\
\text { Sesuvium } \\
\text { portulacastrum } \\
\text { (L.) L./Beldroega } \\
\text { miúda } \\
\end{array}$ & Lvs & & & & $\mathrm{x}$ & & & $\mathrm{x}$ & & $\mathrm{x}$ & $\begin{array}{l}\text { Antimicrobial, antioxidant } \\
\text { (Al-azzawi et al. 2012; } \\
\text { Chandrasekaran et al. } \\
\text { 2011) }\end{array}$ \\
\hline $\begin{array}{l}\text { Alismataceae } \\
\text { Echinodorus } \\
\text { macrophyllus } \\
\text { (Kunth) Micheli/ }^{\text {Chapéu de couro }} \\
\text { Ch }\end{array}$ & Lvs & & & & & & & & & $\mathrm{x}$ & $\begin{array}{l}\text { Anti-inflammatory (Silva } \\
\text { et al. 2016), } \\
\text { nephroprotective (Portella } \\
\text { et al. 2012) }\end{array}$ \\
\hline $\begin{array}{l}\text { Amaranthaceae } \\
\text { Amaranthus } \\
\text { cruentus L./Caruru }\end{array}$ & Lvs & & & & $\mathrm{x}$ & & & $\mathrm{x}$ & & & $\begin{array}{l}\text { Obesity (Kanikowska } \\
\text { et al. 2019), anti- } \\
\text { inflammatory (Tang and } \\
\text { Tsao 2017) }\end{array}$ \\
\hline $\begin{array}{l}\text { Amaranthus } \\
\text { spinosus L./Bledo }\end{array}$ & Lvs & & & & & & & $\mathrm{x}$ & & & $\begin{array}{l}\text { Antioxidant, nutritional, } \\
\text { (Mondal and Maity 2016, } \\
\text { Sarker and Oba 2019), } \\
\text { diabetes (Bavarva and } \\
\text { Narasimhacharya 2013) }\end{array}$ \\
\hline $\begin{array}{l}\text { Amaranthus viridis } \\
\text { L./Bredo } \\
\text { americano }\end{array}$ & Lvs & & & $\mathrm{x}$ & $\mathrm{x}$ & & & $\mathrm{x}$ & & & $\begin{array}{l}\text { Antioxidant, nutritional, } \\
\text { (Sarker and Oba 2019), } \\
\text { Mondal and Maity (2016), } \\
\text { nutritional (Silva et al. } \\
\text { 2018) }\end{array}$ \\
\hline $\begin{array}{l}\text { Anacardiaceae } \\
\text { Spondias tuberosa } \\
\text { Arruda/Ambu }{ }^{1,2}\end{array}$ & Rts & $\mathrm{x}$ & & $\mathrm{x}$ & & & $\mathrm{x}$ & & & $\mathrm{x}$ & $\begin{array}{l}\text { Antimicrobial (Santos } \\
\text { et al. 2019) }\end{array}$ \\
\hline $\begin{array}{l}\text { Aquifoliaceae } \\
\text { Ilex dumosa } \\
\text { Reissek/Mate }\end{array}$ & Lvs & & & & $\mathrm{x}$ & & & & & $\mathrm{x}$ & Gan et al. (2018) \\
\hline $\begin{array}{l}\text { Ilex integerrima } \\
\text { (Vell.) Reissek/ } \\
\text { Mate }^{\mathrm{a}}\end{array}$ & Lvs & & & & & & & & & $\mathrm{x}$ & Gan et al. (2018) \\
\hline $\begin{array}{l}\text { Ilex paraguariensis } \\
\text { A.St.-Hil./Mate }\end{array}$ & Lvs & & & & & & $\mathrm{x}$ & & & $\mathrm{x}$ & $\begin{array}{l}\text { Gan et al. } \\
(2018)+\text { Several other } \\
\text { studies }\end{array}$ \\
\hline $\begin{array}{l}\text { Ilex pseudobuxus } \\
\text { Reissek/Caúna }^{\mathrm{a}}\end{array}$ & Lvs & & & & & & & & & $\mathrm{x}$ & Gan et al. (2018) \\
\hline $\begin{array}{l}\text { Ilex theezans Mart. } \\
\text { ex Reissek/Matea }\end{array}$ & Lvs & & & & & & & & & $\mathrm{x}$ & Gan et al. (2018) \\
\hline $\begin{array}{l}\text { Araceae } \\
\text { Caladium bicolor } \\
\text { (Aiton) Vent./Ara }\end{array}$ & $\begin{array}{l}\text { Lvs, } \\
\text { Rts }\end{array}$ & $\mathrm{x}$ & $\mathrm{x}$ & & & & $\mathrm{x}$ & $\mathrm{x}$ & $\mathrm{x}$ & $\mathrm{x}$ & $\begin{array}{l}\text { Antidiarrheal (Salako } \\
\text { et al. 2015) }\end{array}$ \\
\hline
\end{tabular}


Table 2 (continued)

\begin{tabular}{|c|c|c|c|c|c|c|c|c|c|c|c|}
\hline $\begin{array}{l}\text { Family/species/ } \\
\text { popular names }\end{array}$ & & SO & LI & PI & VE & SP & $\mathrm{CH}$ & PK & $\mathrm{CN}$ & CR & $\begin{array}{l}\text { Correlated studies on } \\
\text { bioactivity }\end{array}$ \\
\hline $\begin{array}{l}\text { Colocasia } \\
\text { esculenta }(\text { L. }) \\
\text { Schott/Inhame }\end{array}$ & Rts & & & & & & & & $\mathrm{x}$ & $\mathrm{x}$ & $\begin{array}{l}\text { Immunomodulator } \\
\text { (Pereira et al. 2018), } \\
\text { diabetes (Eleazu et al. } \\
2016)\end{array}$ \\
\hline $\begin{array}{l}\text { Xanthosoma } \\
\text { sagittifolium (L.) } \\
\text { Schott/Mangará }{ }^{3-4}\end{array}$ & $\begin{array}{l}\text { Lvs, } \\
\text { Rts }\end{array}$ & $\mathrm{x}$ & $\mathrm{x}$ & & & & & $\mathrm{x}$ & & & $\begin{array}{l}\text { Antitumor (Caxito et al. } \\
\text { 2015), prevent colon } \\
\text { cancer (Jackix et al. } \\
\text { 2013), antioxidant } \\
\text { (Arruda et al. 2005), } \\
\text { antifungal (Schmourlo } \\
\text { et al. 2005) }\end{array}$ \\
\hline $\begin{array}{l}\text { Asteraceae } \\
\text { Sonchus oleraceus } \\
\text { L./Serralha lisa }\end{array}$ & Lvs & & & & $\mathrm{x}$ & & $\mathrm{x}$ & & $\mathrm{x}$ & $\mathrm{x}$ & $\begin{array}{l}\text { Antioxidant } \\
\text { (Mawalagedera et al. } \\
\text { 2016); anti-aging agent } \\
\text { (Ou et al. 2015) }\end{array}$ \\
\hline $\begin{array}{l}\text { Ayapana } \\
\text { triplinervis (Vahl) } \\
\text { R.M.King \& } \\
\text { H.Rob./Aiapana }{ }^{1} \text {. }\end{array}$ & Lvs & & & & & & & & & $\mathrm{x}$ & $\begin{array}{l}\text { Antioxidant (Taïlé et al. } \\
\text { 2020) }\end{array}$ \\
\hline $\begin{array}{l}\text { Acmella oleracea } \\
\text { (L.) R.K.Jansen/ } \\
\text { Agrião do Pará }\end{array}$ & Lvs & & & & & & & & $\mathrm{x}$ & $\mathrm{x}$ & $\begin{array}{l}\text { Chemical, nutritional } \\
\text { (Neves et al. 2019), } \\
\text { gastroprotective (Maria- } \\
\text { Ferreira et al. 2014; } \\
\text { Nascimento et al. 2013) }\end{array}$ \\
\hline $\begin{array}{l}\text { Cactaceae } \\
\text { Pereskia aculeata } \\
\text { Mill./Ora pro nobis }\end{array}$ & Lvs & & & & $\mathbf{x}$ & & & & & $\mathrm{x}$ & $\begin{array}{l}\text { Antioxidant, antimicrobial } \\
\text { (Garcia et al. 2019; Souza } \\
\text { et al. 2016), improve } \\
\text { intestinal motility and } \\
\text { lipid profile (Barbalho } \\
\text { et al. 2016) }\end{array}$ \\
\hline $\begin{array}{l}\text { Dioscoreaceae } \\
\text { Dioscorea } \\
\text { chondrocarpa } \\
\text { Griseb./Cará }\end{array}$ & Rts & & & & & & & & & $\mathrm{x}$ & $\begin{array}{l}\text { Antimicrobial (Barnabé } \\
\text { et al. 2014) }\end{array}$ \\
\hline $\begin{array}{l}\text { Dioscorea trifida } \\
\text { L.f./Inhame roxo }\end{array}$ & Rts & & $\mathrm{x}$ & & $\mathrm{x}$ & & & & $\mathrm{x}$ & $\mathrm{x}$ & $\begin{array}{l}\text { Food allergy (Mollica } \\
\text { et al. 2013) }\end{array}$ \\
\hline $\begin{array}{l}\text { Euphorbiaceae } \\
\text { Manihot esculenta } \\
\text { Crantz/Aipim }^{1-16}\end{array}$ & Rts & $\mathrm{x}$ & $\mathrm{x}$ & $\mathrm{x}$ & $\mathrm{x}$ & $\mathrm{x}$ & $\mathrm{x}$ & $\mathrm{x}$ & $\mathrm{x}$ & $\mathrm{x}$ & Several studies \\
\hline $\begin{array}{l}\text { Fabaceae } \\
\text { Neptunia oleracea } \\
\text { Lour./Juquiry } \\
\text { manso }\end{array}$ & Lvs & & & & & & & & $\mathrm{x}$ & $\mathrm{x}$ & $\begin{array}{l}\text { Antioxidant, management } \\
\text { of diabetes (Lee et al. } \\
\text { 2019) }\end{array}$ \\
\hline $\begin{array}{l}\text { Pachyrhizus erosus } \\
\text { (L.) Urb./Jacatupé }\end{array}$ & Rts & & & & & & $\mathrm{x}$ & & & & $\begin{array}{l}\text { Management of blood } \\
\text { glucose (Park et al. 2016, } \\
\text { Santoso et al. 2019) }\end{array}$ \\
\hline
\end{tabular}


Table 2 (continued)

\begin{tabular}{|c|c|c|c|c|c|c|c|c|c|c|c|}
\hline $\begin{array}{l}\text { Family/species/ } \\
\text { popular names }\end{array}$ & & SO & LI & PI & $\mathrm{VE}$ & SP & $\mathrm{CH}$ & PK & $\mathrm{CN}$ & CR & $\begin{array}{l}\text { Correlated studies on } \\
\text { bioactivity }\end{array}$ \\
\hline $\begin{array}{l}\text { Marantaceae } \\
\text { Maranta } \\
\text { arundinacea L./ } \\
\text { Araruta }\end{array}$ & Rts & & & & & $\mathrm{x}$ & & & & \begin{tabular}{l|l}
$\mathrm{x}$ \\
\end{tabular} & $\begin{array}{l}\text { Antiulcerogenic } \\
\text { (Rajashekhara et al. } \\
\text { 2014), immunostimulant } \\
\text { (Kumalasari et al. 2012) }\end{array}$ \\
\hline $\begin{array}{l}\text { Nyctaginaceae } \\
\text { Boerhavia diffusa } \\
\text { L./Tangeraca } \\
\text { mansa }\end{array}$ & Lvs & & & & & & & $\mathrm{x}$ & & & $\begin{array}{l}\text { Gastrointestinal disorders, } \\
\text { anticancer (Mishra et al. } \\
\text { 2014) }\end{array}$ \\
\hline $\begin{array}{l}\text { Boerhavia erecta } \\
\text { L./Erva tostão de } \\
\text { Minas }\end{array}$ & Lvs & & & & & & & $\mathrm{x}$ & & & $\begin{array}{l}\text { Antioxidant, anti- } \\
\text { inflammatory (Compaore } \\
\text { et al. 2018) }\end{array}$ \\
\hline $\begin{array}{l}\text { Oxalidaceae } \\
\text { Oxalis barrelieri } \\
\text { L./Azedinha }\end{array}$ & Lvs & & & & & & & & & $\mathrm{x}$ & $\begin{array}{l}\text { Antidiarrhea (Fokam } \\
\text { Tagne et al. 2018) }\end{array}$ \\
\hline $\begin{array}{l}\text { Piperaceae } \\
\text { Peperomia } \\
\text { pellucida }(\mathrm{L} .) \\
\text { Kunth/ } \\
\text { Jaboti-membeca }\end{array}$ & Lvs & & & & & & & $\mathrm{x}$ & & $\mathrm{x}$ & $\begin{array}{l}\text { Antimicrobial (Alves } \\
\text { et al. 2019) }\end{array}$ \\
\hline $\begin{array}{l}\text { Piper umbellatum } \\
\text { L./Mático }{ }^{1,3,7}\end{array}$ & Lvs & $\mathrm{x}$ & & $x$ & $\mathrm{x}$ & $\mathrm{x}$ & & $\mathrm{x}$ & & $\mathrm{x}$ & $\begin{array}{l}\text { Intestinal anti- } \\
\text { inflammatory } \\
\text { (Arunachalam et al. } \\
\text { 2020), anti-gastric ulcer } \\
\text { (Silva Junior et al. 2016), } \\
\text { anticancer (Iwamoto et al. } \\
\text { 2015) }\end{array}$ \\
\hline $\begin{array}{l}\text { Plumbaginaceae } \\
\text { Plumbago } \\
\text { scandens L./ } \\
\text { Caá-jandiwap, } \\
\text { loco }^{1}\end{array}$ & $\begin{array}{l}\text { Lvs, } \\
\text { Rts }\end{array}$ & & & $\mathrm{x}$ & $\mathrm{x}$ & & $\mathrm{x}$ & & $\mathrm{x}$ & $\mathrm{x}$ & $\begin{array}{l}\text { Antimicrobial } \\
\text { Helicobacter pylori (de } \\
\text { Paiva et al. 2003, Wang } \\
\text { and Huang 2005) }\end{array}$ \\
\hline $\begin{array}{l}\text { Polygonaceae } \\
\text { Rumex acetosella } \\
\text { L./Alecrim de São } \\
\text { José }\end{array}$ & Lvs & & & & & & & $\mathrm{x}$ & & $\mathrm{x}$ & $\begin{array}{l}\text { Management of diabetes } \\
\text { (Özenver et al. 2020) }\end{array}$ \\
\hline $\begin{array}{l}\text { Polygonum } \\
\text { aviculare L./ } \\
\text { Sanguinária }\end{array}$ & Lvs & & & & & & & & & $\mathrm{x}$ & $\begin{array}{l}\text { Pancreatic lipase } \\
\text { inhibitions (Park et al. } \\
\text { 2019), antioxidant, } \\
\text { anti-inflammatory } \\
\text { (Granica et al. 2013), } \\
\text { antiobesity (Sung et al. } \\
\text { 2013), antimicrobial } \\
\text { (Salama and Marraiki } \\
\text { 2010) }\end{array}$ \\
\hline
\end{tabular}


Table 2 (continued)

\begin{tabular}{|c|c|c|c|c|c|c|c|c|c|c|c|}
\hline $\begin{array}{l}\text { Family/species/ } \\
\text { popular names }\end{array}$ & & SO & LI & PI & VE & SP & $\mathrm{CH}$ & PK & $\mathrm{CN}$ & CR & $\begin{array}{l}\text { Correlated studies on } \\
\text { bioactivity }\end{array}$ \\
\hline $\begin{array}{l}\text { Talinaceae } \\
\text { Talinum } \\
\text { paniculatum } \\
\text { (Jacq.) Gaertn./ } \\
\text { Língua de vaca }\end{array}$ & Lvs & & & & & & & & $\mathrm{x}$ & $x$ & $\begin{array}{l}\text { Antimicrobial (Reis et al. } \\
\text { 2015) }\end{array}$ \\
\hline $\begin{array}{l}\text { Tropaoelaceae } \\
\text { Tropaeolum } \\
\text { pentaphyllum } \\
\text { Lam./Capuchinha }^{3}\end{array}$ & $\begin{array}{l}\text { Lvs/ } \\
\text { Rts }\end{array}$ & & & & & & & $\mathrm{x}$ & & $\mathrm{x}$ & $\begin{array}{l}\text { Nutritional, antioxidant } \\
\text { (De Bona et al. 2017), } \\
\text { antimicrobial (Cruz et al. } \\
\text { 2016) }\end{array}$ \\
\hline
\end{tabular}

a Used as tea. Lvs leaves, Rts roots.

\section{Results and Discussion}

Data on the use of Brazilian native plant food species were recovered from books published by nine authors that lived in Brazil during different times in the past five centuries (Table 1). The earliest was the Portuguese Gabriel Soares de Sousa (1540-1592) who lived in in Bahia for 17 years. In his book "Tratado Descritivo do Brasil em 1587" (Descriptive Treaty of Brazil in 1587), he gave detailed information specifically about plants from the Atlantic Forest used by the Amerindians. Friar Cristovão de Lisboa (1583-1652) was a Portuguese who lived in Brazil from 1624 to 1635 and worked in a religious mission in the state of Maranhão; once back in Portugal his observations were published first in 1627 in the book "History of animals and plants of Maranhão" (História dos animais e plantas do Maranhão). As the title suggests, the book has information about animals and plants from the Amazonian region, accompanied by richly detailed high quality drawings which allow identification of the plants (Marques 1996). Also in the seventeenth century, northeast Brazil was invaded by the Dutch, and Guilherme Piso and Marcgrave lived there for 8 years. Later in 1648, back in the Netherlands, they published the book "Historiae Naturalis \& Medicae," in which several native food and medicinal plants used by the Amerindians are described (Piso 1648).

From the Portuguese invasion in 1500 to the next three centuries, Brazilian territory was under strict control in order to prevent natural resources of being exploited by other countries. In 1808, the Portuguese royal family fled to Rio de Janeiro from Portugal after Napoleon Bonaparte's invasion. They lived in Brazil for the next 13 years, a period that was characterized by notable progress in the country's economy, culture and science, such as the creation of the first School of Medicine in Salvador and the Botanical Garden of Rio de Janeiro. Foreigners finally had permission to enter the country and many European naturalists came to Brazil. They explored all regions of the country and identified new plant species and gathered information on their use by the local communities (Brandão et al. 2008a, b; Oliveira et al. 2012). Among these naturalists was the English Richard Spruce (1817-1893), who lived in the Amazon for 17 years. After his death, Wallace edited his "Notes of 
Table 3 Brazilian plant foods not studied and authors who cited their uses

Families/species/popular names/parts used Authors

Alstromeriaceae

Alstroemeria caryophyllaea Jacq./Madressilva/Rts

Alstroemeria monticola Mart. ex Schult. \& Schult.f./

Carajurú/Rts

Bomarea edulis (Tussac) Herb./Jaranganha/Rts

Amaranthaceae

Blutaparon vermiculare (L.) Mears/Caá-ponga/Lvs

Amaryllidaceae

Zephyranthes candida (Lindl.) Herb./Carapitaia/Rts

Anacardiaceae

Spondias venulosa (Engl.) Engl./Acaiá/Rts

Apiaceae

Eryngium nudicaule Lam./Gravatazinho/Rts

Aquifoliaceae

Ilex affinis Gardner/Congonha ${ }^{\mathrm{a}} / \mathrm{Lvs}$

Ilex brasiliensis (Spreng.) Loes./Erva mate ${ }^{\mathrm{a}} / \mathrm{Lvs}$

Ilex conocarpa Reissek/Congonha $/$ Lvs

Ilex chamaedryfolia Reissek/Congonha ${ }^{\mathrm{a}} / \mathrm{Lvs}$

Ilex diuretica Mart. ex Reissek/Congonha $\mathrm{a} / \mathrm{Lvs}$

Ilex paltorioides Reissek/Congonha $\mathrm{a} / \mathrm{Lvs}$

Araceae

Dracontium asperum K.Koch/Taja de cobra/Rts

Urospatha caudata (Poepp.) Schott/Apê/Rts

Xanthosoma riedelianum (Schott) Schott/Mangarito/ Rts

Xanthosoma striatipes (Kunth \& C.D.Bouché)

Madison/Banana do brejo/Rts ${ }^{3}$

Arecaceae

Euterpe oleracea Mart./Açaí/Lvs/Spr 6,7,14

Asteraceae

Erechtites valerianifolius (Wolf) DC./Caruru/Lvs

Pacourina edulis Aubl./Pacurina/Lvs

Balanophoraceae

Langsdorffia hypogaea Mart./Espiga de sangue/Flw

Lophophytum mirabile Schott \& Endl./Batata escamas/Rts

Ombrophytum peruvianum Poepp. \& Endl./Parasita/ Rts

\section{Bignoniaceae}

Dolichandra unguis-cati (L.) L.G.Lohmann/Poampé/ Rts

Campanulaceae

Centropogon cornutus (L.) Druce/Crista de peru/Lvs

\begin{tabular}{l|l|l|l|l|l|l|l|l} 
SO & LI & PI & VE & SP & CH & PK & CN & CR \\
\hline
\end{tabular}

\begin{tabular}{|c|c|c|c|}
\hline & & & $\Lambda$ \\
\hline & & $\mathrm{X}$ & \\
\hline & & $\mathrm{X}$ & $\mathrm{X}$ \\
\hline X & $\mathrm{X}$ & & $\mathrm{X}$ \\
\hline
\end{tabular}

\begin{tabular}{|l|l|l|l|l|l|l|l}
\hline & & & & & $x$ & $x$ & $x$ \\
\hline
\end{tabular}

\begin{tabular}{|l|l|l|l|l|l|l|l|l}
\hline & & & & & $x$ & & $x$ \\
\hline
\end{tabular}


Table 3 (continued)

\begin{tabular}{|c|c|c|c|c|c|c|c|c|c|}
\hline \multirow[b]{2}{*}{ Families/species/popular names/parts used } & \multicolumn{9}{|c|}{ Authors } \\
\hline & $\mathrm{SO}$ & LI & PI & VE & SP & $\mathrm{CH}$ & PK & $\mathrm{CN}$ & CR \\
\hline $\begin{array}{l}\text { Cannaceae } \\
\text { Canna paniculata } \text { Ruiz \& Pav./Paca vira/Rts }\end{array}$ & & & & & & & $\mathrm{x}$ & & $\mathrm{x}$ \\
\hline Canna glauca L./Achira/Rts & & & $\mathrm{x}$ & $\mathrm{x}$ & & $\mathrm{x}$ & $\mathrm{x}$ & $\mathrm{x}$ & $\mathrm{x}$ \\
\hline $\begin{array}{l}\text { Cardiopteridaceae } \\
\text { Citronella paniculata (Mart.) R.A.Howard/ } \\
\text { Congoinha } / \text { Lvs }^{2}\end{array}$ & & & & & & & & & $\mathrm{x}$ \\
\hline $\begin{array}{l}\text { Convolvulaceae } \\
\text { Distimake tuberosus (L.) A.R. Simões \& } \\
\text { Staples/Jetuca/Rts }\end{array}$ & & & & & & & & & $\mathrm{x}$ \\
\hline $\begin{array}{l}\text { Dioscoreaceae } \\
\text { Dioscorea bahiensis Kunth/Cará/Rts }\end{array}$ & & & & & & & & & $\mathrm{x}$ \\
\hline Dioscorea cinnamomifolia Hook./Cará assú/Rts & & & & $\mathrm{x}$ & & & & & $\mathrm{x}$ \\
\hline Dioscorea dodecaneura Vell./Cará barbado/Rts & & & & & & & & & $\mathrm{X}$ \\
\hline Dioscorea fodinarum Kunth/Cará/Rts & & & & & & & & & $\mathrm{x}$ \\
\hline $\begin{array}{l}\text { Dioscorea glandulosa (Klotzsch ex Griseb.) Kunth/ } \\
\text { Cará/Rts }\end{array}$ & & & & & & & & & $\mathrm{x}$ \\
\hline Dioscorea hassleriana Chodat/Cará coco/Rts & & & & $\mathrm{x}$ & & & & & $\mathrm{X}$ \\
\hline Dioscorea heptaneura Vell./Cará branco/Rts & & & & & & & & & $\mathrm{x}$ \\
\hline Dioscorea lacerdaei Griseb./Cará/Rts & & & & & & & & & \\
\hline $\begin{array}{l}\text { Dioscorea laxiflora Mart. ex Griseb./Caratinga bravo/ } \\
\text { Rts }\end{array}$ & & & & & & & & $\mathrm{X}$ & $\mathrm{x}$ \\
\hline Dioscorea leptostachya Gardner/Cará/Rts & & & & & & & & & $\mathrm{x}$ \\
\hline Dioscorea multiflora Mart. ex Griseb./Cará/Rts & & & & & & & & & $\mathrm{x}$ \\
\hline Dioscorea ovata Vell./Cará inhame/Rts & & & & & & & & & $\mathrm{x}$ \\
\hline $\begin{array}{l}\text { Dioscorea piperifolia Humb. \& Bonpl. ex Willd./ } \\
\text { Cará/Rts }\end{array}$ & & & & & & & & $\mathrm{x}$ & $\mathrm{x}$ \\
\hline Dioscorea sinuata Vell./Caratinga brava/Rts & & & & $\mathrm{x}$ & & & & & $\mathrm{x}$ \\
\hline Dioscorea stegelmanniana R.Knuth/Cará/Rts & & & & & & & & & $\mathrm{x}$ \\
\hline Dioscorea trifoliata Kunth/Caranambú/Rts & & & & & & & & & $\mathrm{x}$ \\
\hline $\begin{array}{l}\text { Euphorbiaceae } \\
\text { Manihot caerulescens } \text { Pohl/Maniçoba/Rts }\end{array}$ & & & & & & & & & $\mathrm{x}$ \\
\hline $\begin{array}{l}\text { Manihot caerulescens } \text { Pohl ssp. } \\
\text { caerulescens/Maniçoba/Rts }\end{array}$ & & & & & & & & & $\mathrm{x}$ \\
\hline Manihot dichotoma Ule/Maniçoba/Rts & & & & & & & & & $\mathrm{x}$ \\
\hline Manihot glaziovii Müll.Arg./Maniçoba/Rts & & & & & & & & & $\mathrm{x}$ \\
\hline Manihot violacea Pohl/Maniçoba/Rts & & & & & & & & & $\mathrm{x}$ \\
\hline $\begin{array}{l}\text { Fabaceae } \\
\text { Luetzelburgia auriculata (Allemão) Ducke/Pau } \\
\text { chapada/Rts }\end{array}$ & & & & & & & & & $\mathrm{x}$ \\
\hline $\begin{array}{l}\text { Heliconiaceae } \\
\text { Heliconia psittacorum L.f./Pacová catinga/Rts }\end{array}$ & & & & & & & & & $\mathrm{x}$ \\
\hline $\begin{array}{l}\text { Humiriaceae } \\
\text { Humiria balsamifera (Aubl.) A.St.-Hil./Umiri/Rts }\end{array}$ & & & & & $\mathrm{x}$ & & & $\mathrm{x}$ & \\
\hline
\end{tabular}


Table 3 (continued)

\begin{tabular}{|c|c|c|c|c|c|c|c|c|c|}
\hline \multirow[b]{2}{*}{ Families/species/popular names/parts used } & \multicolumn{9}{|c|}{ Authors } \\
\hline & SO & LI & PI & VE & SP & $\mathrm{CH}$ & PK & $\mathrm{CN}$ & CR \\
\hline Sacoglottis guianensis Benth./Achua/Rts & & & & & & & & $\mathrm{x}$ & \\
\hline $\begin{array}{l}\text { Icacinaceae } \\
\text { Casimirella ampla (Miers) R.A.Howard/Mairá/Rts }\end{array}$ & & & & & & & & $\mathrm{X}$ & $\mathrm{x}$ \\
\hline Leretia cordata Vell./Mata fome/Rts & & & & & & & & & $\mathrm{x}$ \\
\hline $\begin{array}{l}\text { Iridaceae } \\
\text { Phalocallis coelestis (Lehm.) Ravenna/Bibi/Rts }\end{array}$ & & & & & & & & & $\mathrm{x}$ \\
\hline $\begin{array}{l}\text { Malvaceae } \\
\text { Abutilon megapotamicum (Spreng.) A.St.-Hil. \& } \\
\text { Naudin/Benção de Deus/Lvs }\end{array}$ & & & & & & & & & $\mathrm{x}$ \\
\hline $\begin{array}{l}\text { Abutilon purpurascens K.Schum./Benção de Deus/ } \\
\text { Flw }\end{array}$ & & & & & & & & & $\mathrm{x}$ \\
\hline Hibiscus bifurcatus Cav./Algodão do brejo/Rts & & & & & & & & $\mathrm{x}$ & $\mathrm{x}$ \\
\hline $\begin{array}{l}\text { Sphaeralcea bonariensis (Cav.) Griseb./Malvaisco/ } \\
\text { Lvs, Flw }\end{array}$ & & & & & & $\mathrm{x}$ & & & $\mathrm{x}$ \\
\hline $\begin{array}{l}\text { Marantaceae } \\
\text { Goeppertia allouia (Aubl.) Borchs. \& S.Suárez/ } \\
\text { Tupinambor/Rts }\end{array}$ & & & & & & & & & $\mathrm{x}$ \\
\hline $\begin{array}{l}\text { Goeppertia tuberosa (Vell.) Borchs. \& S.Suárez/ } \\
\text { Caeté/Rts }\end{array}$ & & & $\mathrm{x}$ & $\mathrm{x}$ & & & $\mathrm{x}$ & & \\
\hline $\begin{array}{l}\text { Ischnosiphon arouma (Aubl.) Körn./Arumá membeca/ } \\
\text { Rts }\end{array}$ & & & & & & & $\mathrm{x}$ & $\mathrm{X}$ & $\mathrm{x}$ \\
\hline $\begin{array}{l}\text { Ischnosiphon petiolatus (Rudge) L.Andersson/ } \\
\text { Arumá/Rts }\end{array}$ & & & & & & & & $\mathrm{x}$ & \\
\hline Thalia geniculata L./Arumarana/Rts & & & & & & & $\mathrm{x}$ & $\mathrm{x}$ & $\mathrm{x}$ \\
\hline $\begin{array}{l}\text { Nyctaginaceae } \\
\text { Neea theifera } \text { Oerst./Caparrosa } / \text { Lvs }\end{array}$ & & & & & & & & $\mathrm{X}$ & $\mathrm{x}$ \\
\hline $\begin{array}{l}\text { Nymphaeaceae } \\
\text { Victoria amazonica (Poepp.) J.E.Sowerby/Uapé/Rts }\end{array}$ & & & & & & & & $\mathrm{x}$ & \\
\hline $\begin{array}{l}\text { Oxalidaceae } \\
\text { Oxalis hirsutissima Mart. \& Zucc./Azedinha/Lvs }\end{array}$ & & & & & & & & & $\mathrm{x}$ \\
\hline $\begin{array}{l}\text { Phytolaccaceae } \\
\text { Phytolacca rivinoides Kunth \& Bouché/Espinafre } \\
\text { Guiana/Lvs }\end{array}$ & & & & & & & $\mathrm{x}$ & & $\mathrm{x}$ \\
\hline $\begin{array}{l}\text { Phytolacca thyrsiflora Fenzl. ex J.A.Schmidt/Carurú/ } \\
\text { Lvs }\end{array}$ & & & & & & & & & $\mathrm{x}$ \\
\hline $\begin{array}{l}\text { Piperaceae } \\
\text { Peperomia transparens Miq./Língua de sapo/Lvs }\end{array}$ & & & & & & & $\mathrm{x}$ & & \\
\hline $\begin{array}{l}\text { Poaceae } \\
\text { Gynerium sagittatum (Aubl.) P.Beauv./Arina/Spr }\end{array}$ & & & & & & & $\mathrm{x}$ & & \\
\hline $\begin{array}{l}\text { Polygonaceae } \\
\text { Rumex brasiliensis Link/Labaça/Lvs }\end{array}$ & & & & & & & $\mathrm{x}$ & & $\mathrm{X}$ \\
\hline $\begin{array}{l}\text { Portulacaceae } \\
\text { Portulaca halimoides L./Beldroega/Lvs }\end{array}$ & & & & & & & & & $\mathrm{X}$ \\
\hline Portulaca mucronata Link/Beldroega/Lvs & & & & & & & $\mathrm{x}$ & & \\
\hline
\end{tabular}


Table 3 (continued)

\begin{tabular}{|c|c|c|c|c|c|c|c|c|c|}
\hline \multirow[b]{2}{*}{ Families/species/popular names/parts used } & \multicolumn{9}{|c|}{ Authors } \\
\hline & SO & LI & PI & $\mathrm{VE}$ & SP & $\mathrm{CH}$ & PK & $\mathrm{CN}$ & $\mathrm{CR}$ \\
\hline Portulaca pilosa L./Alecrim de São José/Lvs ${ }^{1,2}$ & & & & & & & & & $\mathrm{x}$ \\
\hline $\begin{array}{l}\text { Solanaceae } \\
\text { Solanum commersonii Poir./Batata silvestre/Rts }\end{array}$ & & & & & & & & & $\mathrm{x}$ \\
\hline $\begin{array}{l}\text { Solanum alternatopinnatum Steud./Caruru de } \\
\text { espinho/Lvs }\end{array}$ & & & & $\mathrm{x}$ & & & & & $\mathrm{x}$ \\
\hline $\begin{array}{l}\text { Talinaceae } \\
\text { Talinum fruticosum (L.) Juss./Beldroega grande/Lvs }\end{array}$ & & & & & & & & & $\mathrm{x}$ \\
\hline $\begin{array}{l}\text { Tropaeolaceae } \\
\text { Tropaeolum brasiliense Casar./Capuchinha do Brasil/ } \\
\text { Lvs }\end{array}$ & & & & & & & $\mathrm{x}$ & & $\mathrm{x}$ \\
\hline $\begin{array}{l}\text { Typhaceae } \\
\text { Typha domingensis Pers./Tabua/Rts }\end{array}$ & & & & & & & & $\mathrm{x}$ & $\mathrm{x}$ \\
\hline
\end{tabular}

${ }^{a}$ Used as tea. Lvs leaves, Rts roots, Flw flowers, Spr sprouts.

a botanist in Amazonas and the Andes" (1908), providing them with a bibliographic introduction (Seaward 2000).

It is also important to highlight the efforts of the first Brazilian botanist, Friar José Mariano da Conceição Vellozo. Friar Vellozo was born in 1741 in Minas Gerais and died in Rio de Janeiro, in 1811. From an early age, he was interested in botany. In 1755 he started his religious life in Rio de Janeiro and in 1771 he moved to São Paulo, where he started teaching the indigenous people, simultaneously having the opportunity to learn about the uses and original names of native plants. He was commissioned by the Portuguese Crown to compile an extensive botanical inventory of the Flora in Rio de Janeiro and its surroundings. The result is the work "Florae Fluminensis," completed in 1790, which consists of one volume containing the botanical descriptions of 1639 species of plants, native and exotic, and a further 11 volumes of drawn illustrations. Vellozo was a pioneer in this type of work, and the "Florae" includes valuable information on the use of about 300 plants, including those used as food (Brandão 2019).

The most recent authors cited here studied plants in the nineteenth and early twentieth centuries. The Polish medical doctor P. Chernoviz (1812-1882) and the German pharmacist Theodor Peckolt, (1822-1912), lived in Rio de Janeiro and wrote important books containing information on hundreds of useful and medicinal native plants (Peckolt and Peckolt 2016; Ricardo et al. 2017). In 1947 the French Paul Le Cointe (1870-1956) published the book "Amazônia Brasileira" (Brazilian Amazon), with information on hundreds of plants, their traditional uses, and economic viability. The Portuguese botanist Manoel Pio Corrêa (1874-1934) included description of roots and leaves used as food in his book "Dicionário das plantas úteis do Brasil e das exóticas cultivadas" (Dictionary of Useful Plants of Brazil and Exotic Cultivated), organized in 1926, and his previous work entitled "Flora do Brasil" (Brazilian Flora), published in 1909. Although these authors did their own field work and research, they also declare that they used information present in 
previous sources. We can consider, therefore, that the presence of plants mentioned by previous authors (e.g., Saint-Hilaire, von Martius) is a validation of their uses as food.

A total of 630 plant species used as food were cited in the studied books. Of these, 514 are fruits, most of them already discussed in Teixeira et al. (2019). Thus, in this work, we emphasize the remaining 116 plants, which have other parts used as food. Table 1 shows that Corrêa cited the highest number of species (96) followed by Peckolt (30) and Le Cointe (26). Among the authors that published their works before or during the eighteenth century, Vellozo cited the greatest number of food plants. A total of 18 species was described in his work, while Chernoviz, Piso, Lisboa, and Sousa cited 9, 7, 5, and 5 plants, respectively. Vellozo was Brazilian, and his familiarity with local customs certainly allowed him to have more proximity to the population in order to acquire traditional knowledge. In total, from the works of the 9 authors, 64 roots/tubercles, 52 leaves, 3 flowers, and 2 sprouts were cited as food (Tables 2 and 3).

Among the roots, the use of Manihot esculenta Crantz tubers (aipim, cassava, Euphorbiaceae) was described by all the authors. Cassava tubers are rich in special carbohydrates that release glucose slowly in the body without generating blood glucose spikes (Okafor et al. 2017; Udeme et al. 2015). This composition helps maintain high energy levels for longer, being useful for high performance athletes, and makes this food very suitable for management of diabetes. Cassava is also rich in vitamins A, B1, B2, and C and can be consumed by people and animals. Due to its high benefits, cassava was considered by the Food and Agriculture Organization of the United Nations (FAO/UN) as the most relevant food of the twentieth century (FAO 2013). Other five species of Manihot were also recorded by Corrêa (M. caerulescens Pohl, M. caerulescens Pohl subsp. caerulescens, M. dichotoma Ule, M. glaziovii Müll.Arg., M. violacea Pohl.), but their potential has not been verified to date.

Other useful roots are those from Spondias tuberosa Arruda (Anacardiaceae), Caladium bicolor (Aiton) Vent., and Xanthosoma sagittifolium (L.) Schott (Araceae), cited by different authors along the centuries. They have also been submitted to laboratory studies, which confirmed their health benefits (Table 2). These plants showed antioxidant (Arruda et al. 2005), antimicrobial (Santos et al. 2019; Schmourlo et al. 2005), and antitumoral (Caxito et al. 2015) and could also have the capacity to prevent colon cancer (Kackix et al. 2013). Table 2 shows that roots of Colocasia esculenta (L.) Schott (Araceae) and Maranta arundinacea (Marantaceae) have immunomodulatory activity. Immunomodulators are substances that act directly on the immune system, strengthening its defenses and improving its functions. These effects have been associated to saponins and some types of polysaccharides. The adaptogenic effect of ginsengs roots, for example, is associated with the presence of saponins (Metwaly et al. 2019; Shi et al. 2019), while for mushrooms and seaweed, the effects are associated to saccharides (Ling et al. 2017; Mallard et al. 2019). Saponins and some types of polysaccharides, as starch, are very common in roots, and the effects observed for $C$. esculenta were correlated to the presence of saponins (Azubuike et al. 2018; Eleazu et al. 2016; Pereira et al. 
2018). Other less known edible roots, but also cited along the centuries, are Plumbago scandens L. (Plumbaginaceae), Spondias venulosa (Engl.) Engl. (Anacardiaceae), Canna glauca L. (Cannaceae), and Goeppertia tuberosa (Vell.) Borchs. \& S.Suarez (Marantaceae). The use of roots of $P$. scandens was cited by Piso and Vellozo; P. scandens roots were recently shown to have activity against Helicobacter pylori, what could help in the management of gastric ulcers and prevent cancer (de Paiva et al. 2003; Wang and Huang 2005).

The tubercles of Dioscoreaceae (yams) have been used in the human diet for millenia. Many species have economic importance, due to the presence of diosgenin, which is used as a prototype for oral contraceptive in the pharmaceutical development (Hata et al. 2003). The most important species cultivated for their edible tubers include $D$. alata, originating from Asia, $D$. cayenensis, and $D$. rotundata from West Africa. The tubercles of these species contain several nutrients, including carbohydrates, amino acids, minerals, thiamine, riboflavin, niacin, and ascorbic acid. Diosgenin and allantoin constitute the main secondary bioactive metabolites (Mollica et al. 2013) and have also been shown to possess anti-inflammatory and immunomodulatory properties (Chen et al. 2015). Sixteen species of Dioscorea were cited by Corrêa, and four of them (D. cinnamomifolia Hook., D. hassleriana Chodat, D. sinuata Vell., and D. trifida L.f.) were also registered by Vellozo in the eighteenth century. The use of D. trifida was also observed by Lisboa and Le Cointe in Amazonia. Extracts from the roots were shown to be active in reducing inflammatory parameters associated with food allergies and were suggested to have the potential to prevent and treat this condition (Mollica et al. 2013).

Among plants with leaves that can be used as food, we present 52 species in Tables 2 and 3, with Piper umbellatum L. (Piperaceae) being cited by six among the nine studied authors, including Friar Vellozo. In recent studies this species has shown important results as intestinal anti-inflammatory (Arunachalam et al. 2020), anti-gastric ulcers (Silva Junior et al. 2016), and anticancer (Iwamoto et al. 2015). Other six species were also cited by Vellozo and have shown positive results in studies of bioactivity (Table 2), Sesuvium portulacastrum (L.) L. (Aizoaceae), which has antioxidant and antimicrobial activities (Al-azzawi et al. 2012; Chandrasekaran et al. 2011); Amaranthus cruentus L. (Amaranthaceae) which is anti-inflammatory (Tang and Tsao 2017) and useful in management of obesity (Kanikowska et al. 2019); A. viridis L., which has antioxidant properties and has high nutritional potential (Sarker and Oba 2019; Silva et al. 2018); Sochus oleraceus L. (Asteraceae), that also has activity as antioxidant (Mawalagedera et al. 2016) and as anti-aging agent (Ou et al. 2015); and Pereskia aculeata Mill. (Cactaceae), that has antioxidant and antimicrobial activities (Garcia et al. 2019; Souza et al. 2016) and improves intestinal motility and lipid profile (Barbalho et al. 2016). All these species have been recently considered as non-conventional food plant species ("plantas alimentícias não convencionais - PANCs," in Portuguese). They are plants of spontaneous development, easily found in gardens, backyards, and even on street sidewalks (Kinupp and Lorenzi 2014). Unfortunately, many of the PANC species are considered by the general population as weeds, which is why they are little used in food. Species of PANCs can have significantly higher contents of minerals, fibers, antioxidants, and 
proteins when compared to domesticated plants. The greater quantity of PANCs used today in Brazil corresponds to exotic plants, introduced in the country mainly by the Portuguese and Africans, brought as slaves. It is important to highlight that the plants presented in Tables 2 and 3 are native to Brazil. The species cited especially by the authors from the sixteenth to the eighteenth centuries, were most likely originally domesticated by Amerindians. An example is Pereskia aculeata Mill. (Ora-pro-nobis, Cactaceae, Table 2). Vellozo wrote that “...Africans use ora-pronobis instead of hibiscus...," $P$. aculeata is a native species while many species of hibiscus were introduced in Brazil from Africa. The use of PANCs as food has been highly encouraged in Brazil today and has been adopted by a high percentage of the population, and species present in Tables 1 and 2 are important in this movement due to their historical uses and bioactivities.

Leaves are also frequently used as tea, and the caffeinated tea-like beverages have been very popular all over the world, for centuries. Tea from Camellia sinensis L. (Theaceae), for example, has been consumed for millennia in India, China, and other Asian countries, but became very popular in England in the seventeenth century (Gomes 2019). When the Portuguese royal family came to Brazil in the nineteenth century, there was an interest in the introduction of the $C$. sinensis. Negotiations were made with China and the government brought in some tea seedlings and a few hundred Chinese for their cultivation. This attempt, however, was not successful, as the full development of the plant was not achieved, mainly due to the poor choice of planted species. On the other side, we have mate tea, produced from leaves of the tree Ilex paraguariensis A.St-Hil. (Aquifoliaceae), which has been consumed for millennia by Amerindians living in Argentina, Brazil, Chile, Paraguay, and Uruguay. The Jesuits cultivated mate tea on their missions in Paraguay in the sixteenth century, but formal taxonomic description of the plant, by the French naturalist Auguste de Saint-Hilaire, occurred only in the nineteenth century (Brandão et al. 2012). The French naturalist Aimé Bonpland, who explored the northern parts of South America with Alexander von Humboldt, studied the culture of mate extensively and described the best methods for cultivation, collection and preparation of tea (Corrêa-Filho 1957; Linhares 1969). Currently, mate tea is consumed worldwide as an alternative to coffee and black tea. Studies have demonstrated that leaves of $C$. sinensis and I. paraguariensis have similar chemical characteristics. Both are rich in polyphenols, proteins, enzymes, caffeine, carbohydrates, and inorganics, which provide beneficial properties for health. Several recent studies have confirmed activities as antimicrobial, antioxidant, anti-obesity, antidiabetic, and cardiovascular protective effects (Gan et al. 2018). Data about other ten Ilex species were found in the consulted bibliography (marked with ${ }^{\text {a }}$ in Tables 2 and 3). They show the presence of phenolics and choleretic activity in previous studies (Gan et al. 2018), but have not been investigated in more recent research. The leaves of Echinodorus macrophyllus (Kunth) Micheli (Alismataceae), Ayapana triplinervis (Vahl) R.M.King \& H.Rob. (Asteraceae), Citronella paniculata (Mart.) R.A.Howard (Cardiopteridaceae), and Neea theifera Oerst. (Nyctaginaceae) are also used as tea. Only the first two have been studied and found to have antioxidant activities (Taïlé et al. 2020) and to show anti-inflammatory and nephroprotective 
activities (Portella et al. 2012; Silva et al. 2016). It is interesting to observe that these species belong to different families, and this fact increases the possibility of finding potential bioactive substances with different structures and activities. In addition, three flowers (Langsdorffia hypogaea Mart./Balanophoraceae, Abutilon purpurascens K.Schum, and Sphaeralcea bonariensis (Cav.) Griseb./Malvaceae), and two sprouts (Gynerium sagittatum (Aubl.) P.Beauv./Poaceae and Euterpe oleracea Mart, Arecaceae) were cited by the authors, but the potentials of none of them have been evaluated to date.

The recovery of old eating habits, using different species as food, is considered a strategy for health promotion (Bataglion et al. 2015; Teixeira et al. 2019). Previous studies have shown that phytochemicals, such as phenolic compounds and alkaloids commonly present in plants, can modulate inflammation and improve health problems associated with many degenerative and/or chronic diseases (Oliveira et al. 2014). This has stimulated a renewed interest in accessing plant foods in biodiversity centers, due to the high levels of bioactive substances, capable of preventing such as obesity, diabetes, cancer, coronary heart disease, and Alzheimer's (NeriNuma et al. 2018). The polyphenols present in the leaves, for example, act as antioxidants due to their capacity to stabilize free radicals by breaking the chain of oxidative reactions and/or retarding the formation of free radicals in oxidizable matrices. These processes work as a defense mechanism of the human body, controlling lipid peroxidation and preventing the development of the degenerative diseases. Table 4 shows that 12 plants with antioxidant activity which is correlated with the presence of phenolics. Ou et al. (2015), for example, show the activity of an extract from Sonchus oleraceus L. (Asteraceae) as anti-aging in vitro. In the same

Table 4 Species whose consumption has been suggested to treat or prevent diseases

\begin{tabular}{|c|c|}
\hline Plant with antioxidant effect (leaves) & Correlated diseases \\
\hline Sonchus oleraceus L. & Anti-ageing effect \\
\hline $\begin{array}{l}\text { Amaranthus cruentus L. } \\
\text { Boerhavia erecta } \mathrm{L} . \\
\text { Echinodorus macrophyllus (Kunth) Micheli } \\
\text { Polygonum aviculare L. } \\
\text { Piper umbellatum L. }\end{array}$ & Anti-inflammatory (many diseases) \\
\hline $\begin{array}{l}\text { Piper umbellatum } \mathrm{L} . \\
\text { Xanthosoma sagittifolium (L.) Schott }\end{array}$ & Antitumor/prevent colon cancer \\
\hline $\begin{array}{l}\text { Amaranthus spinosus L. } \\
\text { Colocasia esculenta } \text { (L.) Schott } \\
\text { Neptunia oleracea Lour. }\end{array}$ & Diabetes \\
\hline Pereskia aculeata Mill. & Improves intestinal motility (constipation) \\
\hline $\begin{array}{l}\text { Amaranthus viridis } \mathrm{L} . \\
\text { Pereskia aculeata Mill. } \\
\text { Polygonum aviculare } \mathrm{L} .\end{array}$ & Lipid metabolism (cardiovascular) \\
\hline $\begin{array}{l}\text { Echinodorus macrophyllus (Kunth) Micheli } \\
\text { Sonchus oleraceus L. }\end{array}$ & Nephroprotective \\
\hline $\begin{array}{l}\text { Amaranthus cruentus } \mathrm{L} . \\
\text { Polygonum aviculare L. }\end{array}$ & Obesity \\
\hline
\end{tabular}


way, Carey et al. (2017) have demonstrated that extract from açaí (Euterpe oleracea Mart., Arecaceae) is rich in phenolics, was also effective in prevent age-related cognitive deficits in aged rats. Studies have also shown a positive correlation in the ingestion of antioxidant substances as antidiabetic/antiobesity agents, and this effect occurs by different mechanisms (Cox et al. 2015). Other studies have shown correlation between anti-obesity effects of capsaicin with the presence of flavonoids and polyphenols. The authors have demonstrated that this effect can occur due to changes in the gut microbiota that led to significant impacts on the host's metabolism (Roopchand et al. 2015; Shen et al. 2017; van Dorsten et al. 2012). We can consider that encouraging the consumption of the plants from Table 2 represent an important tool to prevent these diseases, because of their beneficial use for centuries and studies that confirm their relevance as functional foods. We also argue that species from Table 3, and all other cited in historical documents, should have priority in the studies on bioactivity for developing biological products. Detailed, critical studies are essential in order to validate - or invalidate - claims concerning bioactivity and specificity, so that those plants with beneficial properties can be better exploited.

Brazil has the most diverse flora of the world, with 38,700 species approximately, which corresponds to $26.5 \%$ of the total of known species (Ulloa et al. 2017). The plants listed in this study confirm the great variety of native plant foods supplied by the Brazilian flora. The data recovered from this historical review covered four of the six main phytogeographic domains of the country: Atlantic Forest, Cerrado, Caatinga, and Amazonia. This information is also important because it was recorded in a time that Brazil's vegetation was still preserved, and the interactions between the Amerindians and the new inhabitants were frequent. The commercialization and management of these plants can also contribute to biodiversity conservation and improve the income of local populations.

\section{Conclusion}

In this study we collected written references to the traditional use of leaves and roots of native Brazilian plant species and summarize their suggested bioactive properties. These plants may have a great potential for use as functional foods, despite most of them being unknown to the population and to science. The valorization of biodiversity, through sustainable use of underexploited species, is a way to slow down the erosion of genetic diversity in remote regions, while studies with these plants can lead to development of innovative bio-products with high commercial value. We argue that incentives for better use of the species listed in this study must be considered and they should be included in agricultural programs that are done throughout the country.

Acknowledgments The authors are grateful to Conselho Nacional de Desenvolvimentos Científico e Tecnológico (CNPq) and Fundação de Amparo a Pesquisas de Minas Gerais 
(FAPEMIG) for financial support in the development of Dataplamt. MGLB is funded by CNPq with a research fellowship (310389/2017-8). We are also in debt to Prof. Christine Clayton from University of Heidelberg for her help with text review.

\section{References}

Al-azzawi A, Alguboori A, Hachim MY et al (2012) Preliminary phytochemical and antibacterial screening of Sesuvium portulacastrum in the United Arab Emirates. Pharm Res 4:219-224. https://doi.org/10.4103/0974-8490.102269

Alves NSF, Setzer WN, da Silva JKR (2019) The chemistry and biological activities of Peperomia pellucida (Piperaceae): a critical review. J Ethnopharmacol 232:90-102. https://doi. org/10.1016/j.jep.2018.12.021

Arruda S, Souza EMT, Siqueira E (2005) Carotenoids from malanga (Xanthosoma Sagittifolium) leaves protect cells against oxidative stress in rats. Int J Vitam Nutr Res 75:161-168. https:// doi.org/10.1024/0300-9831.75.2.161

Arunachalam K, Damazo AS, Macho A et al (2020) Piper umbellatum L. (Piperaceae): phytochemical profiles of the hydroethanolic leaf extract and intestinal anti-inflammatory mechanisms on 2,4,6 trinitrobenzene sulfonic acid induced ulcerative colitis in rats. J Ethnopharmacol 254:112707. https://doi.org/10.1016/j.jep.2020.112707

Azubuike NC, Onwukwe OS, Onyemelukwe AO et al (2018) Impact of Colocasia esculenta extract and fractions on high-fat diet-induced changes in body weight, adipose tissue and liver of rats. Pak J Pharm Sci 31:2149-2156

Barbalho SM, Guiguer ÉL, Marinelli PS et al (2016) Pereskia aculeata miller flour: metabolic effects and composition. J Med Food 19:890-894. https://doi.org/10.1089/jmf.2016.0052

Barnabé M, Saraceni CHC, Dutra-Correa M, Suffredini IB (2014) The influence of Brazilian plant extracts on Streptococcus mutans biofilm. J Appl Oral Sci 22:366-372. https://doi. org/10.1590/1678-775720140085

Bataglion GA, da Silva FMA, Eberlin MN, Koolen HHF (2015) Determination of the phenolic composition from Brazilian tropical fruits by UHPLC-MS/MS. Food Chem 180:280-287. https://doi.org/10.1016/j.foodchem.2015.02.059

Bavarva JH, Narasimhacharya AV (2013) Systematic study to evaluate anti-diabetic potential of Amaranthus spinosus on type-1 and type-2 diabetes. Cell Mol Biol (Noisy-le-Grand) 59:OL1818-OL1825

Bockmann FA, Rodrigues MT, Kohsldorf T et al (2018) Brazil's government attacks biodiversity. Science 360:865. https://doi.org/10.1126/science.aat7540

Brandão MGL (2019) Plantas úteis e medicinais nas obras de Frei Vellozo. 3i Editora, Belo Horizonte

Brandão MGL, Zanetti NNS, Oliveira GR et al (2008a) Plantas medicinais da Estrada Real. MG Biota 1:4-15

Brandão MGL, Zanetti NNS, Oliveira P et al (2008b) Brazilian medicinal plants described by 19th century European naturalists and in the Official Pharmacopoeia. J Ethnopharmacol 120:141-148. https://doi.org/10.1016/j.jep.2008.08.004

Brandão MGL, Grael CF, Fagg CW (2011) European naturalists and medicinal plants of Brazil. In: Biological diversity and sustainable resources use. InTech, pp 101-120

Brandão MGL, Pignal M, Romaniuc S et al (2012) Useful Brazilian plants listed in the field books of the French naturalist Auguste de SaintHilaire (1779-1853). J Ethnopharmacol 143:488-500. https://doi.org/10.1016/j.jep.2012.06.052

Breitbach UB, Niehues M, Lopes NP et al (2013) Amazonian Brazilian medicinal plants described by C.F.P. von Martius in the 19th century. J Ethnopharmacol 147:180-189. https://doi. org/10.1016/j.jep.2013.02.030 
Carey AN, Miller MG, Fisher DR et al (2017) Dietary supplementation with the polyphenol-rich açaí pulps (Euterpe oleracea Mart. and Euterpe precatoria Mart.) improves cognition in aged rats and attenuates inflammatory signaling in BV-2 microglial cells. Nutr Neurosci 20:238-245. https://doi.org/10.1080/1028415X.2015.1115213

Caxito MLC, Correia RR, Gomes ACC et al (2015) In Vitro Antileukemic Activity of Xanthosoma sagittifolium (Taioba) Leaf Extract. Evid Based Complement Alternat Med 2015:1-10. https:// doi.org/10.1155/2015/384267

Chandrasekaran M, Senthilkumar A, Venkatesalu V (2011) Fatty acid methyl esters from the leaves of Sesuvium portulacastrum L. Eur Rev Med Pharmacol 15:775

Chen Y, Tang YM, Yu SL et al (2015) Advances in the pharmacological activities and mechanisms of diosgenin. Chin J Nat Med 13:578-587. https://doi.org/10.1016/S1875-5364(15)30053-4

Compaore M, Bakasso S, Meda R et al (2018) Antioxidant and anti-inflammatory activities of fractions from Bidens engleri O.E. Schulz (Asteraceae) and Boerhavia erecta L. (Nyctaginaceae). Medicines 5:53. https://doi.org/10.3390/medicines5020053

Corrêa-Filho V (1957) Ervais e do Brasil ervateiros. Ministério da Agricultura, Rio de Janeiro

Cox AJ, West NP, Cripps AW (2015) Obesity, inflammation, and the gut microbiota. Lancet Diabetes Endocrinol 3:207-215. https://doi.org/10.1016/S2213-8587(14)70134-2

Cruz R, Denardi L, Mossmann N et al (2016) Antimicrobial activity and chromatographic analysis of extracts from Tropaeolum pentaphyllum lam. Tubers. Molecules 21:566. https://doi. org/10.3390/molecules21050566

de Almeida Jackix E, Monteiro EB, Raposo HF, Vanzela EC, Amaya-Farfán J (2013) Taioba (Xanthosoma sagittifolium) Leaves: Nutrient Composition and Physiological Effects on Healthy Rats. J Food Sci 78:1929-1934. https://doi.org/10.1111/1750-3841.12301

De Bona GS, Boschetti W, Bortolin RC et al (2017) Characterization of dietary constituents and antioxidant capacity of Tropaeolum pentaphyllum Lam. J Food Sci Technol 54:3587-3597. https://doi.org/10.1007/s13197-017-2817-z

de Paiva SR, Figueiredo MR, Aragão TV, Kaplan MAC (2003) Antimicrobial activity in vitro of plumbagin isolated from Plumbago species. Mem Inst Oswaldo Cruz 98:959-961. https://doi. org/10.1590/S0074-02762003000700017

Dean WA (1996) A ferro e fogo: a história da devastação da Mata Attântica brasileira. Companhia das Letras, Rio de Janeiro, $\mathrm{p} 484$

Domingues HMB, Kleiche-Dray M, Petitjean P (2012) Historia das substâncias naturais: saberes tradicionais e quimica: Amazônia e América Latina. Museu de Astronomia e Ciências Afins/ Institut de Recher chepourle Développement, Rio de Janeiro/Paris

Eleazu CO, Eleazu KC, Iroaganachi MA (2016) Effect of cocoyam (Colocasia esculenta), unripe plantain (Musa paradisiaca) or their combination on glycated hemoglobin, lipogenic enzymes, and lipid metabolism of streptozotocin-induced diabetic rats. Pharm Biol 54(1):91-97. https:// doi.org/10.3109/13880209.2015.1016181

Fagg CW, Lughadha EN, Milliken W et al (2015) Useful Brazilian plants listed in the manuscripts and publications of the Scottish medic and naturalist George Gardner (1812-1849). J Ethnopharmacol 161:18-29. https://doi.org/10.1016/j.jep.2014.11.035

FAO (2013) Cassava's huge potential as 21st Century crop. http://www.fao.org/news/story/en/ item/176780/icode/. Accessed 10 Aug 2020

Fernandes TM (2004) Plantas Medicinais: memória da ciência no Brasil. Editora Fiocruz, Rio de Janeiro

Ferrão JEM, Caixinhas ML, Liberato MC (2008) A ecologia, as plantas e a interculturalidade. Percursos de Interculturalidade, Portugal

Fokam Tagne MA, Noubissi PA, Fankem GO, Kamgang R (2018) Effects of Oxalis barrelieri L. (Oxalidaceae) aqueous extract on diarrhea induced by Shigella dysenteriae type 1 in rats. Heal Sci Rep 1:e20. https://doi.org/10.1002/hsr2.20

Gan RY, Zhang D, Wang M, Corke H (2018) Health benefits of bioactive compounds from the genus Ilex, a source of traditional caffeinated beverages. Nutrients 10:1682. https://doi. org/10.3390/nu10111682 
Garcia JAA, Corrêa RCG, Barros L et al (2019) Phytochemical profile and biological activities of "Ora-pro-nobis" leaves (Pereskia aculeata Miller), an underexploited superfood from the Brazilian Atlantic Forest. Food Chem 294:302-308. https://doi.org/10.1016/j. foodchem.2019.05.074

Gomes L (2019) Escravidão: Do Primeiro Leilão de Cativos em Portugal até a Morte de Zumbi dos Palmares. Globo Livros, Rio de Janeiro

Granica S, Czerwińska ME, Żyżyńska-Granica B, Kiss AK (2013) Antioxidant and antiinflammatory flavonol glucuronides from Polygonum aviculare L. Fitoterapia 91:180-188. https://doi.org/10.1016/j.fitote.2013.08.026

Hata Y, Reguero MT, Garcia LA, Buitrago G, Alvarez A (2003) Evaluación del contenido de sapogeninas em variedades nativas de inhame (Dioscorea spp.) provenientes de la coleccion de La Universidad del Córdoba. Rev Colomb 32:149-157

Iwamoto LH, Vendramini-Costa DB, Monteiro PA et al (2015) Anticancer and anti-inflammatory activities of a standardized dichloromethane extract from Piper umbellatum L. leaves. Evid Based Complement Alternat Med 2015:1-8. https://doi.org/10.1155/2015/948737

Jackix EA, Monteiro EB, Raposo HF et al (2013) Taioba (Xanthosoma sagittifolium) leaves: nutrient composition and physiological effects on healthy rats. J Food Sci 78:H1929-H1934. https://doi.org/10.1111/1750-3841.12301

Kahleova H, Levin S, Barnard N (2017) Cardio-metabolic benefits of plant-based diets. Nutrients 9:848. https://doi.org/10.3390/nu9080848

Kanikowska D, Kanikowska A, Rutkowski R et al (2019) Amaranth (Amaranthus cruentus L.) and canola (Brassica napus L.) oil impact on the oxidative metabolism of neutrophils in the obese patients. Pharm Biol 57:140-144. https://doi.org/10.1080/13880209.2019.1569696

Kinupp WK, Lorenzi H (2014) Plantas Alimentícias Não Convencionais (PANC) no Brasil. Instituto Plantarum, Nova Odessa

Kumalasari ID, Harmayani E, Lestari LA et al (2012) Evaluation of immunostimulatory effect of the arrowroot (Maranta arundinacea. L) in vitro and in vivo. Cytotechnology 64:131-137. https://doi.org/10.1007/s10616-011-9403-4

Lee SY, Mediani A, Ismail IS et al (2019) Antioxidants and $\alpha$-glucosidase inhibitors from Neptunia oleracea fractions using 1H NMR-based metabolomics approach and UHPLC-MS/MS analysis. BMC Complement Altern Med 19:7. https://doi.org/10.1186/s12906-018-2413-4

Ling N, Zhou X, Ji Y, Li W, Ji C, Qi Z (2017) Immuno-modulatory and cellular antioxidant activities of $\kappa$-selenocarrageenan in combination with Epirubicin in $\mathrm{H} 22$ hepatoma-bearing mice. Biomed Pharmacother 91:132-137. https://doi.org/10.1016/j.biopha.2017.04.064

Linhares T (1969) História econômica do mate. Livraria José Olympio, Rio de Janeiro

Maezumi SY, Alves D, Robinson M, Souza JG, Levis G, Barnett RL, Oliveira EA, Urrego D, Schaan D (2018) Iriarte JR (2018) The legacy of 4,500 years polyculture agroforestry in the eastern Amazon. Nature 4:540-547

Mallard B, Leach DN, Wohlmuth H, Tiralongo J (2019) Synergistic immuno-modulatory activity in human macrophages of a medicinal mushroom formulation consisting of Reishi, Shiitake and Maitake. PLoS One 14(11):e0224740. https://doi.org/10.1371/journal.pone.0224740

Maria-Ferreira D, da Silva LM, Mendes DA, Cabrini Dde A, Nascimento AM, Iacomini M, Cipriani TR, Santos AR, Werner MF, Baggio CH (2014) Rhamnogalacturonan from Acmella oleracea (L.) R.K. Jansen: gastroprotective and ulcer healing properties in rats. PLoS One 9(1):e84762. https://doi.org/10.1371/journal.pone.0084762

Marques JF (1996) Frei Cristóvão de Lisboa, missionário no Maranhão e Grão Pará (1624-1635) e a defesa dos índios brasileiros. Rev da Fac Let 13:323-351

Mawalagedera SMMR, Ou Z-Q, McDowell A, Gould KS (2016) Effects of boiling and in vitro gastrointestinal digestion on the antioxidant activity of Sonchus oleraceus leaves. Food Funct 7:1515-1522. https://doi.org/10.1039/C5FO01508A

Melina V, Craig W, Levin S (2016) Position of the academy of nutrition and dietetics: vegetarian diets. J Acad Nutr Diet 116:1970-1980. https://doi.org/10.1016/j.jand.2016.09.025 
Metwaly AM, Lianlian Z, Luqi H, Deqiang D (2019) Black ginseng and its saponins: preparation, phytochemistry and pharmacological effects. Molecules 24(10):1856. https://doi.org/10.3390/ molecules24101856

Mishra S, Aeri V, Gaur PK, Jachak SM (2014) Phytochemical, therapeutic, and ethnopharmacological overview for a traditionally important herb: boerhavia diffusa Linn. Biomed Res Int 2014:1-19. https://doi.org/10.1155/2014/808302

Mollica JQ, Cara DC, D'Auriol M et al (2013) Anti-inflammatory activity of American yam Dioscorea trifida L.f. in food allergy induced by ovalbumin in mice. J Funct Foods 5:1975-1984. https://doi.org/10.1016/j.jff.2013.09.020

Mondal A, Maity TK (2016) Antibacterial activity of a novel fatty acid (14E, 18E, 22E, 26E)-methyl nonacosa-14, 18, 22, 26 tetraenoate isolated from Amaranthus spinosus. Pharm Biol 54:2364-2367. https://doi.org/10.3109/13880209.2016.1155628

Mügge FL, Paula-Souza J, Melo JC, Brandão MG (2016) Native plant species with economic value from Minas Gerais and Goiás: a discussion on the currentness of the data recovered by the French naturalist Auguste de Saint-Hilaire. Hortic Bras 34:455-462. https://doi.org/10.1590/ s0102-053620160402

Nascimento AM, de Souza LM, Baggio CH, Werner MF, Maria-Ferreira D, da Silva LM, Sassaki GL, Gorin PA, Iacomini M, Cipriani TR (2013) Gastroprotective effect and structure of a rhamnogalacturonan from Acmella oleracea. Phytochemistry 85:137-142. https://doi.org/10.1016/j. phytochem.2012.08.024

Neri-Numa IA, Soriano Sancho RA, Pereira APA, Pastore GM (2018) Small Brazilian wild fruits: nutrients, bioactive compounds, healthpromotion properties and commercial interest. Food Res Int 103:345-360. https://doi.org/10.1016/j.foodres.2017.10.053

Neves DA, Schmiele M, Pallone JAL et al (2019) Chemical and nutritional characterization of raw and hydrothermal processed jambu (Acmella oleracea (L.) R.K. Jansen). Food Res Int 116:1144-1152. https://doi.org/10.1016/j.foodres.2018.09.060

Okafor EN, Erukainure OL, Ozumba AU et al (2017) Cassava flour substitution modulates Glycemic responses and Glycemic index of wheat breads in apparent healthy volunteers. J Diet Suppl 14:446-452. https://doi.org/10.1080/19390211.2016.1267061

Oliveira VB, Yamada LT, Fagg CW, Brandão MGL (2012) Native foods from Brazilian biodiversity as a source of bioactive compounds. Food Res Int 48:170-179. https://doi.org/10.1016/j. foodres.2012.03.011

Oliveira VB, Ferreira AVM, Oliveira MC, Teixeira MM, Brandão MGL (2014) Effects of Xylopia aromatica (Lam.) Mart. fruit on metabolic and inflammatory dysfunction induced by high refined carbohydrate-containing-diet in mice. Food Res Int 62:541-550. https://doi. org/10.1016/j.foodres.2014.03.066

Ou ZQ, Rades T, McDowell A (2015) Anti-ageing effects of Sonchus oleraceus L. (pūhā) leaf extracts on H2O2-induced cell senescence. Molecules 20:4548-4564. https://doi.org/10.3390/ molecules 20034548

Özenver N, Güvenalp Z, Kuruüzüm-Uz A, Demirezer LÖ (2020) Inhibitory potential on key enzymes relevant to type II diabetes mellitus and antioxidant properties of the various extracts and phytochemical constituents from Rumex acetosella L. J Food Biochem 44(10):e13415. https://doi.org/10.1111/jfbc.13415

Park CJ, Lee H-A, Han J-S (2016) Jicama (Pachyrhizus erosus) extract increases insulin sensitivity and regulates hepatic glucose in C57BL/Ksjdb/db mice. J Clin Biochem Nutr 58:56-63. https://doi.org/10.3164/jcbn.15-59

Park JY, Kim CS, Park K-M, Chang P-S (2019) Inhibitory characteristics of flavonol-3-Oglycosides from Polygonum aviculare L. (common knotgrass) against porcine pancreatic lipase. Sci Rep 9:18080. https://doi.org/10.1038/s41598-019-54546-8

Peckolt T, Peckolt G (2016) História das Plantas Medicinais e Úteis do Brasil. Fino Traço, Belo Horizonte 
Pereira PR, Corrêa ACNTF, Vericimo MA, Paschoalin VMF (2018) Tarin, a potential Immunomodulator and COX-inhibitor lectin found in Taro (Colocasia esculenta). Compr Rev Food Sci Food Saf 17:878-891. https://doi.org/10.1111/1541-4337.12358

Piso G (1648) História Natural e Médica da Índia Ocidental. Tradução Mário Lôbo Leal, Rio de Janeiro: Ministério da Educação e Cultura. Instituto Nacional do Livro; 1957. 682p.

Portella VG, Cosenza GP, Diniz LRL et al (2012) Nephroprotective Effect of Echinodorus macrophyllus Micheli on Gentamicin-Induced Nephrotoxicity in Rats. Nephron Extra 2:177-183. https://doi.org/10.1159/000339181

Rajashekhara N, Ashok B, Sharma P, Ravishankar B (2014) Evaluation of acute toxicity and antiulcerogenic study of rhizome starch of two source plants of Tugaksheeree (Curcuma angustifolia Roxb. and Maranta arundinacea Linn.). AYU (An Int Q J Res Ayurveda) 35:433. https:// doi.org/10.4103/0974-8520.159013

Reis LFC, Cerdeira CD, Paula BF et al (2015) Chemical characterization and evaluation of antibacterial, antifungal, antimycobacterial, and cytotoxic activities of Talinum paniculatum. Rev Inst Med Trop Sao Paulo 57:397-405. https://doi.org/10.1590/S0036-46652015000500005

Ricardo LM, de Paula-Souza J, Andrade A, Brandão MGL (2017) Plants from the Brazilian Traditional Medicine: species from the books of the Polish physician Piotr Czerniewicz (Pedro Luiz Napoleão Chernoviz, 1812-1881). Rev Bras Farmacogn 27:388-400. https://doi. org/10.1016/j.bjp.2017.01.002

Roopchand DE, Carmody RN, Kuhn P et al (2015) Dietary Polyphenols Promote Growth of the Gut Bacterium Akkermansia muciniphila and Attenuate High-Fat Diet-Induced Metabolic Syndrome. Diabetes 64:2847-2858. https://doi.org/10.2337/db14-1916

Salako OA, Akindele AJ, Shitta OM et al (2015) Antidiarrhoeal activity of aqueous leaf extract of Caladium bicolor (Araceae) and its possible mechanisms of action. J Ethnopharmacol 176:225-231. https://doi.org/10.1016/j.jep.2015.10.035

Salama HMH, Marraiki N (2010) Antimicrobial activity and phytochemical analyses of Polygonum aviculare L. (Polygonaceae), naturally growing in Egypt. Saudi J Biol Sci 17:57-63. https:// doi.org/10.1016/j.sjbs.2009.12.009

Santos ATL, Pereira Carneiro JN, Pereira da Cruz R et al (2019) UPLC-MS-ESI-QTOF analysis and antifungal activity of the Spondias tuberosa Arruda leaf and root hydroalcoholic extracts. Antibiotics 8:240. https://doi.org/10.3390/antibiotics8040240

Santos-Fonseca DJ, Coelho-Ferreira M, Fonseca-Kruel V (2019) Useful plants referenced by the naturalista Richar Spruce in the 19th century in the state of Pará, Brazil. Acta Bot Brasilica 33(2):221-231. https://doi.org/10.1590/0102-33062018abb0344

Santoso P, Amelia A, Rahayu R (2019) Jicama (Pachyrhizus erosus) fiber prevents excessive blood glucose and body weight increase without affecting food intake in mice fed with high-sugar diet. J Adv Vet Anim Res 6:222. https://doi.org/10.5455/javar.2019.f336

Sarker U, Oba S (2019) Nutraceuticals, antioxidant pigments, and phytochemicals in the leaves of Amaranthus spinosus and Amaranthus viridis weedy species. Sci Rep 9:20413. https://doi. org/10.1038/s41598-019-50977-5

Schmourlo G, Mendonça-Filho RR, Alviano CS, Costa SS (2005) Screening of antifungal agents using ethanol precipitation and bioautography of medicinal and food plants. J Ethnopharmacol 96:563-568. https://doi.org/10.1016/j.jep.2004.10.007

Seaward MRD (2000) Richard Spruce, botânico e desbravador da América do Sul. História, Ciências, Saúde-Manguinhos 7:379-390. https://doi.org/10.1590/S0104-59702000000300007

Shen W, Shen M, Zhao X et al (2017) Anti-obesity effect of capsaicin in mice fed with high-fat diet is associated with an increase in population of the gut bacterium akkermansia muciniphila. Front Microbiol 8:272. https://doi.org/10.3389/fmicb.2017.00272

Shi ZY, Zeng JZ, Wong (2019) Chemical structures and pharmacological profiles of ginseng saponins. Molecules 24(13):2443. https://doi.org/10.3390/molecules24132443. PMID: 31277214

Silva Junior IF, Balogun SO, de Oliveira RG et al (2016) Piper umbellatum L.: a medicinal plant with gastric-ulcer protective and ulcer healing effects in experimental rodent models. J Ethnopharmacol 192:123-131. https://doi.org/10.1016/j.jep.2016.07.011 
Silva GP, Fernandes DC, Vigliano MV et al (2016) Flavonoid-enriched fraction from Echinodorus macrophyllus aqueous extract exhibits high in-vitro and in-vivo anti-inflammatory activity. $\mathrm{J}$ Pharm Pharmacol 68:1584-1596. https://doi.org/10.1111/jphp.12620

Silva LFLE, Souza DC, Resende LV et al (2018) Nutritional evaluation of nonconventional vegetables in Brazil. An Acad Bras Cienc 90:1775-1787. https://doi. org/10.1590/0001-3765201820170509

Sousa GS (1587) Tratado Descritivo do Brasil em 1587. São Paulo: Companhia Editora Nacional; 1987. 389p.

Souza L, Caputo L, Inchausti De Barros I et al (2016) Pereskia aculeata Muller (Cactaceae) leaves: chemical composition and biological activities. Int J Mol Sci 17:1478. https://doi.org/10.3390/ ijms 17091478

Sung YY, Yoon T, Yang WK, Kim SJ, Kim DS, Kim HK (2013) The antiobesity effect of polygonum aviculare 1. ethanol extract in high-fat diet-induced obese mice. Evid Based Complement Alternat Med 2013:626397. https://doi.org/10.1155/2013/626397

Taïlé J, Arcambal A, Clerc P et al (2020) Medicinal plant polyphenols attenuate oxidative stress and improve inflammatory and vasoactive markers in cerebral endothelial cells during Hyperglycemic condition. Antioxidants 9:573. https://doi.org/10.3390/antiox9070573

Tang Y, Tsao R (2017) Phytochemicals in quinoa and amaranth grains and their antioxidant, antiinflammatory, and potential health beneficial effects: a review. Mol Nutr Food Res 61:1600767. https://doi.org/10.1002/mnfr.201600767

Teixeira N, Melo JCS, Batista LF et al (2019) Edible fruits from Brazilian biodiversity: a review on their sensorial characteristics versus bioactivity as tool to select research. Food Res Int 119:325-348. https://doi.org/10.1016/j.foodres.2019.01.058

Udeme N, Okafor P, Eleazu C (2015) The metabolic effects of consumption of yellow cassava (Manihot esculenta Crantz) on some biochemical parameters in experimental rats. Int J Toxicol 34:559-564. https://doi.org/10.1177/1091581815606085

Ulloa UC, Acevedo-Rodríguez P, Beck S et al (2017) An integrated assessment of the vascular plant species of the Americas. Science 358:1614-1617. https://doi.org/10.1126/science.aao0398

Valli M, Russo HM, Bolzani VS (2018) The potential contribution of the natural products from Brazilian biodiversity to bioeconomy. An Acad Bras Cienc 90:763-778. https://doi. org/10.1590/0001-3765201820170653

van Dorsten FA, Peters S, Gross G et al (2012) Gut microbial metabolism of polyphenols from black tea and red wine/grape juice is SourceSpecific and colon-region dependent. J Agric Food Chem 60:11331-11342. https://doi.org/10.1021/jf303165w

Venâncio MD, Pope K, Sieber S (2018) Brazil's new government threatens food security and biodiversity. Nature 564:39-39. https://doi.org/10.1038/d41586-018-07611-7

Wang YC, Huang T-L (2005) Screening of anti-Helicobacter pylori herbs deriving from Taiwanese folk medicinal plants. FEMS Immunol Med Microbiol 43:295-300. https://doi.org/10.1016/j. femsim.2004.09.00 